\title{
Outcomes of Australian Patients Receiving Non-funded Anti-PD-1 Immune Checkpoint Inhibitors for Non-melanoma Cancers
}

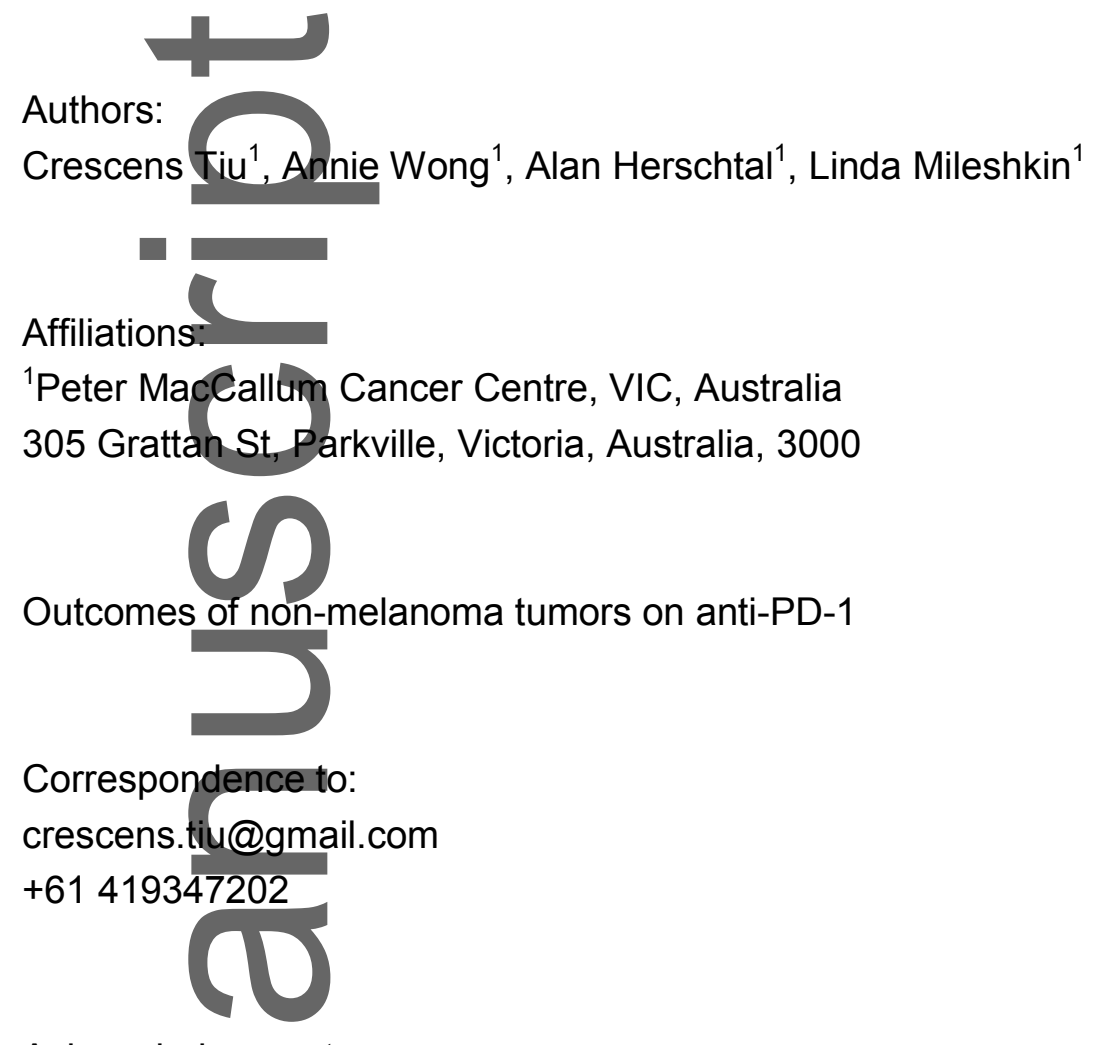

Acknowledgements:

The authors would like to thank Bristol-Myers Squibb for permission to publish information of patients on the nivolumab access scheme.

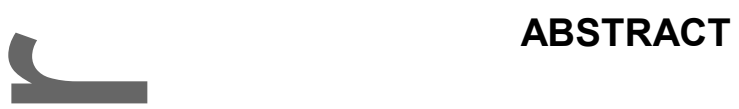

Aim: To characterize the outcomes of patients with non-melanoma solid tumors receiving anti-PD-1 immunotherapy not funded by the Australian Pharmaceutical Benefits Scheme.

Methods: Medical records of patients with metastatic non-melanoma tumor diagnoses treated with anti-PD-1 (self-funded pembrolizumab or nivolumab through an access program) from 1 January 2014 to 31 December 2016 at Peter MacCallum Cancer Centre were retrospectively reviewed. Events after 31 December 2016 were censored.

Results: Of 47 patients identified, 27 (57\%) had lung cancer. Twenty-six had compassionate access to nivolumab (24 lung, 1 renal, 1 gastroesophageal with possible new lung primary). Median overall survival was 5.7 months. Eleven (23\%) achieved a partial response; none had complete response. 20 (43\%) had disease progression on first imaging; 16 (48\%) of these continued treatment beyond radiological progression, with 3 achieving subsequent partial responses. Ten $(21 \%)$ were not re-staged mostly due to rapid deterioration or death.

This is the author manuscript accepted for publication and has undergone full peer review but has not been through the copyediting, typesetting, pagination and proofreading process, which may lead to differences between this version and the Version of Record. Please cite this article as doi:

10.1111/ajco.12867.

This article is protected by copyright. All rights reserved. 
At 6 months and 12 months, $9(20 \%)$ and $2(4 \%)$ remained on treatment, respectively. Five $(12 \%)$ discontinued treatment due to immune-related toxicities. Of 34 patients who died, $71 \%$ received treatment within the last month of life; $38 \%$ died in an acute hospital. None of 25 patients with poor Eastern Cooperative Oncology Group performance scores of 2-4 responded.

Conclusion: The response rates and overall survival of patients with NSCLC, renal carcinoma and triple negative breast cancer of good performance status receiving anti-PD-1 therapy outside of a clinical trial are consistent with clinical trial data. However, patients with poor ECOG performance status are unlikely to respond. Careful patient selection and counseling about the potential outcomes of self-funding treatment in this setting is needed.

Keywords inivolumab, patient selection, PD-1, pembrolizumab, performance status

\section{BACKGROUND}

\section{PD-1 inhibitors for advanced non-melanoma tumors}

PD-1 inhibitors have shown encouraging activity in a range of non-melanoma solid tumors. For example, nivolumab has been shown to be superior to chemotherapy for patients who have received prior platinum-based therapy for metastatic non-small cell lung cancer (NSCLC). Median overall survivals (OS) were 9.2 and 12.2 months for squamous and nonsquamous NSCLC, respectively. Response rates were around $20 \%$. Subgroup analyses showed an association between PDL-1 expression and treatment efficacy for non-squamous NSCLC $(1,2)$. This led to its approval under the Therapeutic Goods Australia (TGA). Until recently, patients have only been able to receive nivolumab through enrolment into an "early patient access" (EPA) or "compassionate use" program for investigational drugs, through Bristol-Myers Squibb. On 1 August 2017, nivolumab was made available on PBS for second line treatment of advanced NSCLC, in patients with World Health Organization (WHO) performance status of 0-1. Pembrolizumab was shown to improve OS for previously-treated PDL-1 positive advanced NSCLC, compared to docetaxel (3). However, it is not on PBS for this indication.

In patients with advanced renal cell carcinoma (RCC) who have received prior antiangiogenic therapy, nivolumab demonstrated prolonged median OS of 24 months, with fewer toxicities compared to everolimus. Its benefit was observed irrespective of PDL-1 expression (4). This led to its TGA approval, and was available on an EPA program for a period of time. On 1 August 2017, it was made available on PBS for second line treatment of $\mathrm{RCC}$, in patients with WHO performance status of $\leq 2$.

In a phase II trial, pembrolizumab demonstrated activity in heavily pre-treated metastatic triple-negative breast cancer (TNBC), with an objective response rate of $5 \%$, regardless of PD-L1 expression (5). Preliminary data suggested promising results for its use as first-line therapy in PD-L1 positive metastatic TNBC (6). 
Other tumor types that have shown response to anti-PD-1 and PDL-1 agents include metastatic refractory urothelial carcinoma, head and neck squamous cell carcinoma (SCC), cervical SCC and Merkel cell carcinoma (7-9). Numerous other trials are currently underway, testing various tumor types and clinical settings, with increasing focus on trying to find accurate predictive biomarkers such as PD-L1 expression, tumor-infiltrating lymphocytes, mutational burden and immune gene signatures (10).

\section{Self-funding of anti-PD-1 treatment and patient selection}

The encouraging outcomes of these trials have raised substantial public interest in immunotherapy as a therapeutic option in oncology. As a result, patients with advanced nonmelanoma solid tumors, having exhausted all standard options, are increasingly requesting treatment with self-funded anti-PD-1 therapy. Out-of-pocket costs for patients range from around Australian $\$ 4750$ to $\$ 7000$ per cycle (US $\$ 3600$ to $\$ 5400)(11,12)$.

However, patients enrolled in clinical trials have required excellent performance status and organ function, and may not be reflective of patients seen in routine clinical practice. Hence, there is a lack of real world efficacy and toxicity data for patients receiving anti-PD-1 in a non-trial setting.

Wong et al. reviewed the experience of 91 melanoma patients receiving checkpoint inhibitor therapy at Peter-MacCallum Cancer Centre, and found that patients with poor Eastern Cooperative Oncology Group (ECOG) scores of 2-3 were unlikely to respond, with an OS of only 1.8 months (13).

Lomax ef al. reviewed a small cohort of patients $(n=21)$ who received self-funded pembrolizumab at Chris O'Brien Lifehouse in New South Wales, Australia. 13 (62\%) had ECOG score of 2-4. The majority of the patients in the study had diagnoses of mesothelioma and sarcoma. Patients who requested treatment had worse outcomes compared to those who were offered treatment by their clinician. Only $3(17 \%)$ of the 18 treated patients achieved partial response (PR) while none had complete response (CR). Three patients died before receiving any dose and four died within 13-43 days of receiving only one dose (14).

Therefore, the aim of this study was to characterize the outcomes of patients with nonmelanoma solid tumors receiving anti-PD-1 checkpoint inhibitors in a non-trial and non-PBSfunded setting at a comprehensive cancer center.

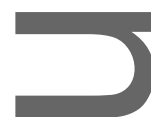

\section{METHODS}

Medical records of patients with metastatic non-melanoma tumor diagnoses treated with anti-PD-1, either self-funded pembrolizumab or nivolumab through an access program, from 1 January 2014 to 31 December 2016, at our comprehensive cancer center, were retrospectively reviewed. Data collected included information about patient characteristics, treatment outcomes, and quality of care indicators. The latter comprised of continuing cancer treatment near time of death, inpatient hospital and intensive care unit admissions near end 
of life, involvement with palliative care and evidence of advanced care planning (15). Events after 31 December 2016 were censored.

All statistical analyses were performed in the $\mathrm{R}$ statistical software package version 3.3.1 using standard and validated statistical procedures. Survival curves were estimated using the Kaplan-Meier method. The study protocol and data collected were approved by our institution's ethics committee.

Patient characteristics

\section{RESULTS}

A total of 47 eligible patients were identified. Table 1 outlines the patient characteristics. Mean age was 62 years; 55\% were male. 27 (57\%) had NSCLC. Twenty-two (46\%) had good ECOG scores of 0-1.

Twenty-six (55\%) received compassionate access to nivolumab. This included patients with NSCLC (24), renal cell carcinoma (1), and gastroesophageal cancer with possible new lung primary (1). The rest self-funded pembrolizumab. Median follow-up was 4.1 months.

Number of lines of prior palliative systemic treatment ranged from 0-7. Thirty-five patients (75\%) received localized palliative radiotherapy at some point during the course of anti-PD-1 treatment. $7(15 \%)$ received steroids shortly prior to immunotherapy or were on a weaning dose for indications such as central nervous system metastases, spinal cord compression, bronchial-obstruction and asthma. None had pre-existing autoimmune disease and no patients had testing for PDL-1 expression on their tumor prior to therapy.

\section{Treatment responses and overall survival}

Ten patients (21\%) were not re-staged on treatment mostly due to rapid deterioration or death. The median duration of treatment was 2.1 months. At 6 months and 12 months, 9 $(20 \%)$ and $2(4 \%)$ remained on treatment, respectively.

The overall response rate was $23 \%$. Eleven $(23 \%)$ achieved a PR but none had a CR. All responders had tumor types of only either lung, renal or TNBC. Median OS was 5.7 months.

The response rate of patients who received nivolumab was $27 \%$ with median OS of 6.3 months. Patients who self-funded pembrolizumab had a slightly lower response rate of $19 \%$ and OS of 4.14 months. None of the 25 patients with poor ECOG scores of 2-4 responded (Table 2).

OS at 3 months and 6 months were $62 \%$ and $49 \%$, respectively (Figure 1). PFS at 3 months and 6 months were $34 \%$ and $21 \%$, respectively (Figure 2 ). 
Thirty-four patients died as of 31 December 2016. Causes of death were mainly due to progressive cancer (20) and sepsis (3). One patient died of aspiration, gastrointestinal bleed and pulmonary embolism, while another died of functional decline post spinal surgery. Causes of death for 9 patients were not documented, but no patient appeared to have died from auto-immune toxicity.

\section{Treatment decisions at first radiologic progression}

Of 33 patients who had radiologic disease progression at initial re-staging, 11 patients changed treatment. Six patients (18\%) then received best supportive care.

Sixteen (48\%), however, still continued with anti-PD-1. Two of these were according to patients' wishes, both of whom were self-funding pembrolizumab. Other documented reasons for continuing included limited treatment options available for the patient; the patient seemed clinically stable or better despite the radiologic progression; or that there was a plan to add other forms of therapy onto the anti-PD-1. Upon continuing treatment, 3 patients eventually had radiologic PR (median duration of response of 2.4 months). Patient 1 had NSCLC and received additional radiotherapy to disease in the lung resulting in a subsequent PR after 1,4 months. Patient 2 received self-funded pembrolizumab for NSCLC and received concurrent radiotherapy to brain and lung metastases, plus erlotinib, an epidermal growth factor receptor (EGFR) inhibitor. Patient 3 had RCC and achieved PR 2 months after initial radiologic progression, which on hindsight was likely pseudoprogression.

\section{Safety and treatment discontinuation}

A total of $8(17 \%)$ immune-related adverse events occurred, 4 of which required the patient to discontinue treatment (Grade 3 pneumonitis $=2$, Grade 3 hepatitis=1, Grade 4 hypophysitis=1). The rest had Grade 1-2 hypothyroidism and pneumonitis which was treated with thyroxine replacement and steroids, respectively.

Of 42 patients who stopped treatment before 31 December 2016, reasons cited for discontinuation included death $(17 \%)$, disease progression $(52 \%)$, clinical deterioration without radiologic progression $(12 \%)$ and treatment toxicity $(9.5 \%)$. Other reasons $(9.5 \%)$ included care transferred to another facility, severe cellulitis, and functional decline post spinal surgery following a fall.

\section{Quality of care indicators}

Within the last month of life, 24 of $33(71 \%)$ patients still received some form of anti-cancer treatment, and $21(62 \%)$ were admitted to hospital, including 4 to an intensive care unit (ICU). These 4 patients had ECOG scores of 2-3 and only 2 of them had advanced care plans (ACP) in place. More patients died in an acute hospital, than at home or hospice $(38 \%$, versus $8 \%$, versus $6 \%$, respectively). 
The overall rate of palliative care referrals for all patients was reasonably high at $70 \%$. The overall rate for having an ACP in place was $47 \%$. Of the 34 patients who died, $27(80 \%)$ had palliative care involvement, and $18(53 \%)$ had an ACP.

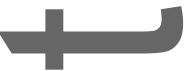

The following two cases demonstrate the contrasting outcomes for self-funding patients, with performance status and tumor type as the most likely predictive factors:

1. A 59-year-old woman, ECOG 1, with metastatic EGFR-mutant NSCLC, after 3 lines of prior treatment, received self-funded pembrolizumab for a total of 17.7 months, despite radiologic progression after 4 months of commencing treatment. Her clinician allowed her to receive concurrent erlotinib, an EGFR inhibitor, then subsequently, stereotaetic brain radiotherapy, whole brain radiotherapy, and palliative radiotherapy to the lung, all the while continuing pembrolizumab. She had her last dose of pembrolizumab 3 months prior to death as a result of functional decline after spinal surgery following a fall.

2. A 67-year-old man, ECOG 3, with metastatic NSCLC, self-funded one dose of pembrolizumab, had clinical decline and radiologic progression 11 days after, but still decided to pay for another dose before transferring to hospice 4 days later. He died 37 days after the first dose of pembrolizumab.

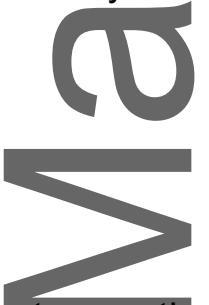

\section{DISCUSSION}

In this retrospective study, tumor type and performance status seem to be important predictors of clinical benefit from anti-PD-1 therapy. We highlight some factors that may influence and challenge the clinicians' decision-making process when providing patients with self-funded treatment.

The response rates and OS results of the tumor types which have the most evidence for anti-PD-1 benefit, namely lung, renal and TNBC, were comparable to published trial data. The sole renal cancer patient on nivolumab had ongoing PR after 9.7 months as of 31 December 2016. However, no patients receiving self-funded pembrolizumab for other tumor types appeared to respond.

Importantly, all the patients who achieved PR had a good ECOG of $0-1$, which is a selection criteria formost randomized controlled trials. Indeed, the patients with ECOG 3-4 who selffunded pembrolizumab only received a total of 1-2 doses and died not long afterwards, which is consistent with findings in our previously published melanoma cohort (13). This implies that ECOG performance status is an important predictor for response in this setting and should be used for better patient selection and counseling.

The majority of patients in our series had a diagnosis of NSCLC and mostly receiving nivolumab via a compassionate access program. Patients eligible for this program were more likely to commence anti-PD-1 treatment after fewer lines of treatment than those who were self-funding therapy. This could be reflective of the influence of financial costs on a

This article is protected by copyright. All rights reserved. 
patient's treatment decisions. Patients with limited resources might be more likely choose a therapy that is freely available than the expensive one whose efficacy is still under investigation for their particular tumor type. When and how a clinician presents the option of self-funded treatment to a patient is also likely to influence their treatment pathway.

Deciding whether to continue treatment in the event of disease progression, especially if a patient has the means to self-fund therapy, is an ongoing challenge for clinicians. It can be difficult to differentiate true progression from pseudoprogression, a phenomenon where there is initial increase in size or number of tumor lesions, but is then followed by a delayed response. However, the rate of pseudo-progression with PD-1 inhibitors is generally low and hence it is not recommended to continue treatment in a patient who is not symptomatically stable $(16$,

Interestingly, the addition of targeted radiotherapy while continuing immunotherapy resulted in eventual response in two of our patients. It is possible that this led to an abscopal effect to out-of-target tumor sites.

The rate of palliative care involvement in the case of these patients was encouraging although not 100 percent. This is likely reflective of the acknowledgement of the importance of good palliative care for patients at our cancer center. ACP documentation in our electronic medical record may not have been completely representative of the actual number of patients who had ACPs already in place. A clinician can enter onto the database that an ACP discussion has taken place, however one is not required to fill in all the details or scan the ACP. Moreover, the settings in which discussions around ACP and resuscitation wishes have occurred are unclear, although anecdotally these tend to happen during an acute hospital admission rather than as an outpatient. Whether these could have affected decisions about self-funding, cessation of treatment and place of death is unknown.

It was not consistently documented in the records whether self-funding treatment was requested by the patient or offered by the clinician, and therefore difficult to assess whether there would have been a difference in outcome as was observed by Lomax et al (14). Nevertheless, careful discussion regarding the risks and benefits must be tailored to individual patients, with respect for both patient's and clinician's autonomy.

Limitations of the study include its retrospective nature and small sample size, with only small numbers of less common tumor types. However, the finding that patients with poor performance status are unlikely to respond to immunotherapy prescribed for advanced, multiple relapsed disease, regardless of tumor type is an important finding for oncologists, given that discussions with patients about unfunded anti-cancer drugs are now occurring frequently in daily practice. For example, a recent survey of members of the Medical Oncology Group of Australia showed that $92 \%$ of respondents had discussed and $68 \%$ had prescribed at least one unfunded anticancer drug in the last 3 months (18). We also eagerly await the availability of more robust biomarkers that can accurately predict patients who will respond to PD-1 inhibitors. In Australia, PDL-1 expression testing has only started to become more available, and this may prove useful in selecting patients, particularly those with NSCLC, who would benefit from self-funding pembrolizumab. 


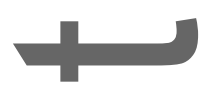

\section{CONCLUSION}

The response rates and overall survival of patients with NSCLC, renal carcinoma and triple negative breast cancer of good performance status receiving anti-PD-1 therapy outside of a clinical trial are consistent with clinical trial data. However, patients with poor ECOG performance status are unlikely to respond. Careful patient selection and counseling about the potential outcomes of self-funding treatment in this setting is needed.

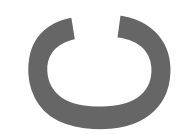

\section{ACKNOWLEDGEMENTS}

The authors would like to thank Bristol-Myers Squibb, for permission to publish information of patients on the nivolumab access scheme.

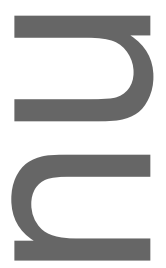

\section{REFERENCES}

(1) Brahmer J,Reckamp KL, Baas P, Crinò L, Eberhardt WEE, Poddubskaya E, et al. Nivolumab versus Docetaxel in Advanced Squamous-Cell Non-Small-Cell Lung Cancer. The New England Journal of Medicine 2015 Jul 9,;373(2):123-135.

(2) BorghaeiH, Paz-Ares L, Horn L, Spigel DR, Steins M, Ready NE, et al. Nivolumab versus Docetaxel in Advanced Nonsquamous Non-Small-Cell Lung Cancer. The New England Journal of Medicine 2015 Oct 22,;373(17):1627-1639.

(3) Herbst RS, Baas P, Kim D, Felip E, Pérez-Gracia J,L., Han J, et al. Pembrolizumab versus docetaxel for previously treated, PD-L1-positive, advanced non-small-cell lung cancer (KEYNOTE-010): a randomised controlled trial. The Lancet ;387(10027):1540-1550.

(4) Motzer RJ, Escudier B, McDermott DF, George S, Hammers HJ, Srinivas S, et al. Nivolumab versus Everolimus in Advanced Renal-Cell Carcinoma. The New England Journal of Medicine 2015 Nov 5,;373(19):1803-1813.

(5) Adams S, Schmid P, Rugo H. Phase 2 study of pembrolizumab monotherapy for previously treated metastatic triple-negative breast cancer: KEYNOTE-086 cohort A. Journal of Clinical Oncology 2017;35.

(6) Adams S, Loi S, Toppmeyer D, Cescon DW. Phase 2 study of pembrolizumab as first-line therapy for PD-L1-positive metastatic triple-negative breast cancer: Preliminary data from KEYNOTE-086 cohort B. Journal of Clinical Oncology $2017 ; 35$.

(7) Gupta S, Gill D, Poole A, Agarwal N. Systemic Immunotherapy for Urothelial Cancer: Current Trends and Future Directions. Cancers 2017 Jan 1,;9(2):15. 
(8) Economopoulou P, Perisanidis C, Giotakis EI, Psyrri A. The emerging role of immunotherapy in head and neck squamous cell carcinoma (HNSCC): anti-tumor immunity and clinical applications. Annals of translational medicine 2016

May;4(9):173.

(9) Frenel J, Le Tourneau C, O'Neil BH, Ott PA, Piha-Paul S, Gomez-Roca C, et al. Pembrolizumab in patients with advanced cervical squamous cell cancer:

Preliminary results from the phase Ib KEYNOTE-028 study. JCO 2016;34(15):5515.

(10) Gibney GT, Weiner LM, Atkins MB. Predictive biomarkers for checkpoint inhibitor-based immunotherapy. Lancet Oncology 2016 Dec 1,;17(12):e551.

(11) Melanoma Metastatic Nivolumab. 2017; Available at:

https://www.eviq.org.au/Protocol/tabid/66/categoryid/415/id/1728/Melanoma+Metast atic+Nivolumab+.aspx. Accessed Jun 12, 2017.

(12) Melanoma Metastatic Pembrolizumab. 2017; Available at: https://www.eviq.org.au/Protocol/tabid/66/categoryid/415/id/1729/Melanoma+Metast atic+Pembrolizumab.aspx. Accessed Jun 12, 2017.

(13) Wong A, Williams M, Milne D, Morris K, Lau P, Spruyt O, et al. Performance status as a predictor of response to anti-PD1 for metastatic melanoma. Asia Pacific Journal of Clinical Oncology 2016 Aug,;12(Suppl. 4):71.

(14) Lomax AJ, Beith J, Bhadri V, Boyer M, Grimison P, Horvath LG, et al. Outcomes of patients with non- melanoma solid tumours receiving self- funded pembrolizumab at Chris O'Brien-Lifehouse. Internal Medicine Journal 2016 Dec;46(12):1392-1398.

(15) Earle CC, Park ER, Lai B, Weeks JC, Ayanian JZ, Block S. Identifying Potential Indicators of the Quality of End-of-Life Cancer Care From Administrative Data. JCO 2003;21(6):1133-1138.

(16) Nishino M, Dahlberg SE, Adeni AE, Lydon CA, Hatabu H, Jänne PA, et al. Tumor Response Dynamics of Advanced Non-small cell Lung Cancer Patients Treated with PD-1 Inhibitors: Imaging Markers for Treatment Outcome. Clin Cancer Res 2017

(17) Kim HK, Heo MH, Lee HS, Sun J, Lee S, Ahn JS, et al. Comparison of RECIST to immune-related response criteria in patients with non-small cell lung cancer treated with immune-checkpoint inhibitors. Cancer Chemother Pharmacol 2017;80(3):591-598.

(18) Karikios DJ, Mileshkin L, Martin A, Ferraro D, Stockler MR. Discussing and prescribing expensive unfunded anticancer drugs in Australia. ESMO Open 2017;2(2):e000170. 
TABLES

Table 1. Patient and tumor characteristics

\begin{tabular}{|c|c|c|c|c|}
\hline & & All & $\begin{array}{c}\text { Compassionate } \\
\text { access to } \\
\text { nivolumab }\end{array}$ & $\begin{array}{c}\text { Self-funded } \\
\text { pembrolizumab }\end{array}$ \\
\hline$\square$ & & 47 & $26(55 \%)$ & $21(45 \%)$ \\
\hline Age & Median [range] & $64[31-81]$ & $62.5[32-80]$ & $63[43-80]$ \\
\hline \multirow[t]{2}{*}{ Sex } & Male & $26(55 \%)$ & 15 & 11 \\
\hline & Female & $21(45 \%)$ & 11 & 10 \\
\hline \multirow{5}{*}{$\begin{array}{l}\text { Lines of prior } \\
\text { palliative systemic } \\
\text { treatment }\end{array}$} & 0 & $5(11 \%)$ & 4 & 1 \\
\hline & 1 & $17(36 \%)$ & 12 & 5 \\
\hline & 2 & $10(21 \%)$ & 6 & 4 \\
\hline & 3 & $9(19 \%)$ & 3 & 6 \\
\hline & $\geq 4$ & $6(12 \%)$ & 1 & 5 \\
\hline \multirow[t]{9}{*}{ Tumor types } & NSCLC† & $27(57 \%)$ & 24 & 3 \\
\hline & Breast & $4(9 \%)$ & 0 & 4 \\
\hline & Head and Neck $\ddagger$ & $4(9 \%)$ & 0 & 4 \\
\hline & Renal cell carcinoma & $3(9 \%)$ & 1 & 2 \\
\hline & Urothelial carcinoma & $2(4 \%)$ & 0 & 2 \\
\hline & Upper gastrointestinal & $2(4 \%)$ & 1 & 1 \\
\hline & Gynaecologic§ & $2(4 \%)$ & 0 & 2 \\
\hline & Mesothelioma & $2(4 \%)$ & 0 & 2 \\
\hline & $\begin{array}{l}\text { Pancreas } \\
\text { neuroendocrine } \\
\text { tumour }\end{array}$ & $1(2 \%)$ & 0 & 1 \\
\hline \multirow{2}{*}{$\begin{array}{l}\text { Steroid use prior to } \\
\text { treatment }\end{array}$} & No & $40(85 \%)$ & 22 & 18 \\
\hline & Yes & $7(15 \%)$ & 4 & 3 \\
\hline \multirow{5}{*}{ ECOG score } & 0 & $3(6 \%)$ & 3 & 0 \\
\hline & 1 & $19(40 \%)$ & 10 & 9 \\
\hline & 2 & $20(43 \%)$ & 11 & 9 \\
\hline & 3 & $4(9 \%)$ & 2 & 2 \\
\hline & 4 & $1(2 \%)$ & 0 & 1 \\
\hline
\end{tabular}

†SCC (5), adenocarcinoma (22)

łoral SCC (1), tongue SCC (1), tonsil SCC (1), parotid adenocarcinoma (1) §cervix SCC (1), high-grade serous ovarian (1) 
Table 2. Response rates and median overall survival according to tumor type, ECOG score and anti-PD-1 treatment

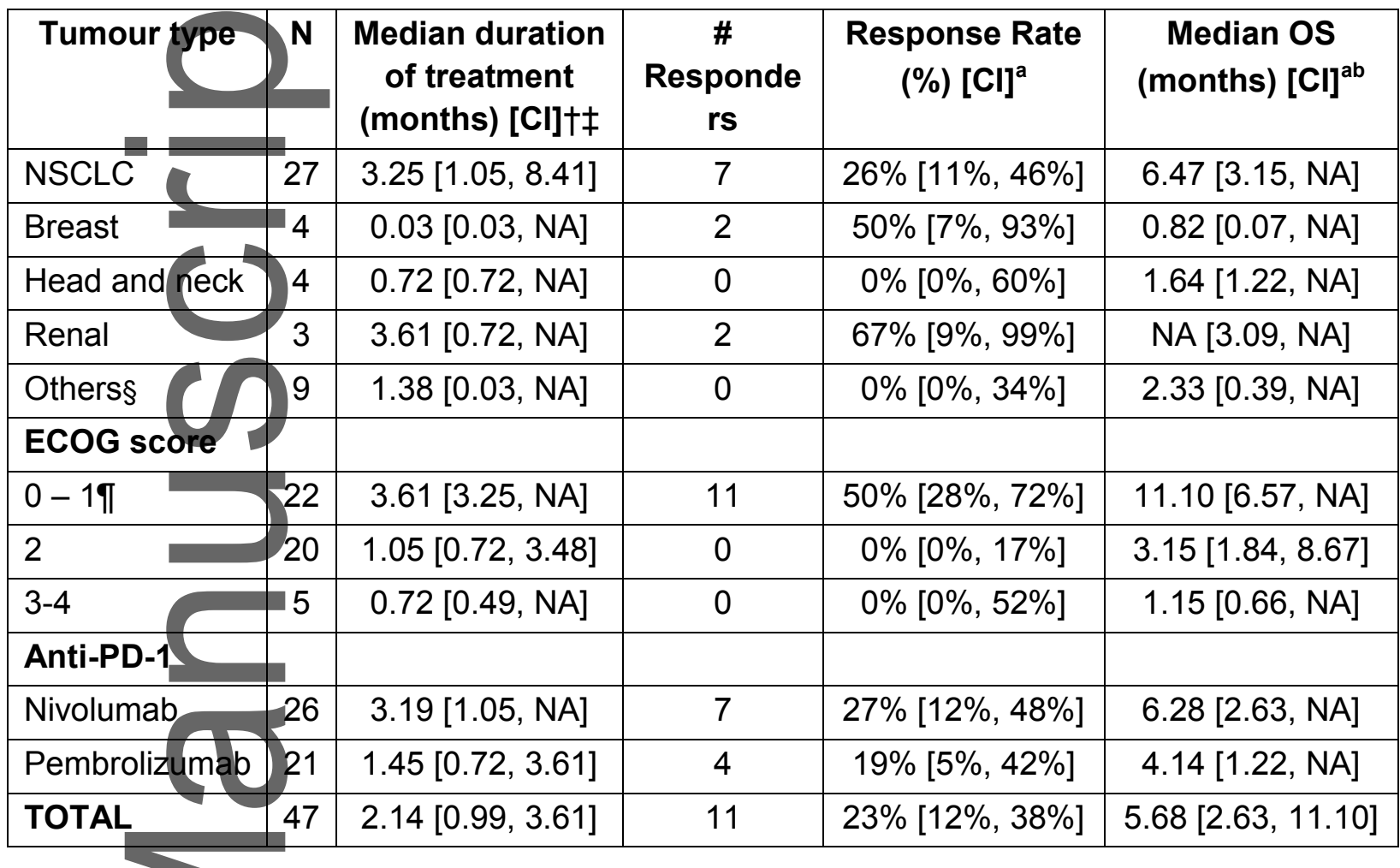

† Cl: $95 \%$ confidence interval.

‡ NA: Could not be estimated due to insufficient events.

$\S$ Consists of patients with urothelial (2), gastroesophageal (2), gynecological (2), mesothelioma (2), and pancreatic neuroendocrine tumor (1).

đConsists of patients with lung (14), renal (3), breast (3), gastroesophageal (1), head \& neck (1).

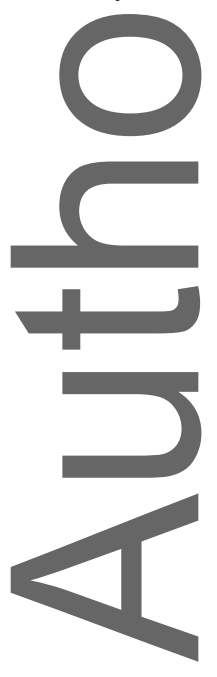




\section{FIGURE LEGENDS}

Figure 1. Kaplan-Meier curve for overall survival. 3-month OS 62\% [49\%, 77\%]; 6-month OS $49 \%[37 \%, 66 \%]$.

FIGURES

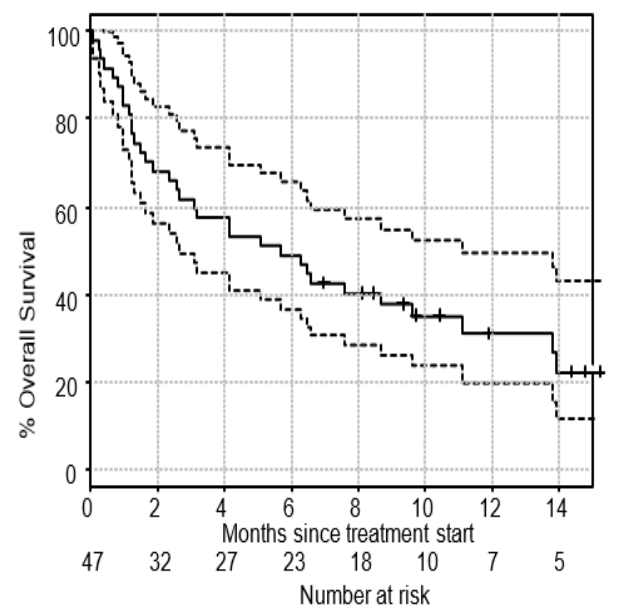

Figure 1. Kaplan-Meier curve for overall survival. 3-month OS $62 \%$ [49\%, 77\%]; 6-month OS $49 \%$ [37\%, 66\%].
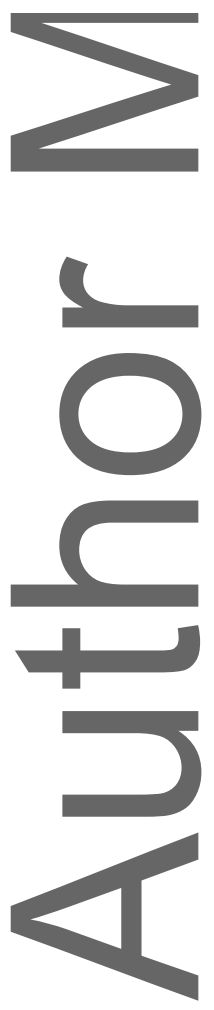

This article is protected by copyright. All rights reserved. 
Figure 2. Kaplan-Meier curve for progression-free survival. 3-month PFS 34\% [23\%, 51\%]; 6-month PFS $21 \%[12 \%, 37 \%]$.
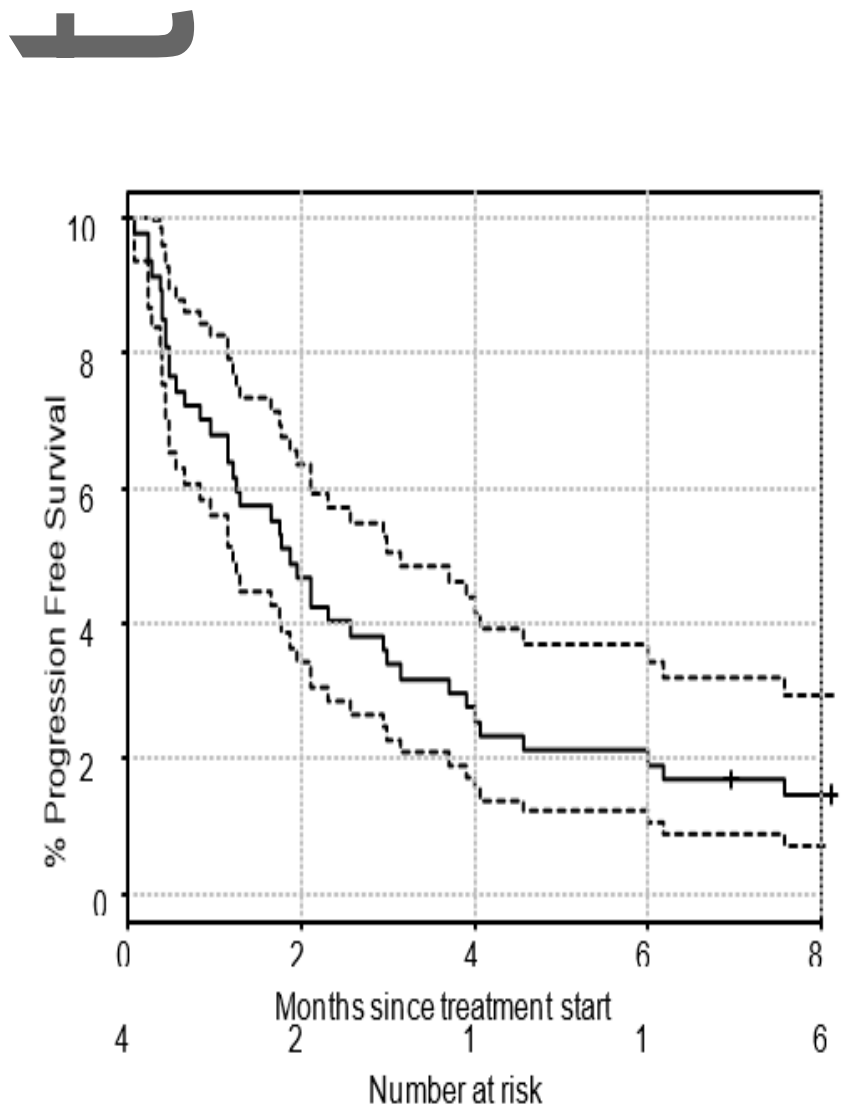

Figure 2. Kaplan-Meier curve for progression-free survival. 3-month PFS 34\% [23\%, 51\%]; 6-month PFS 21\% [12\%,37\%].

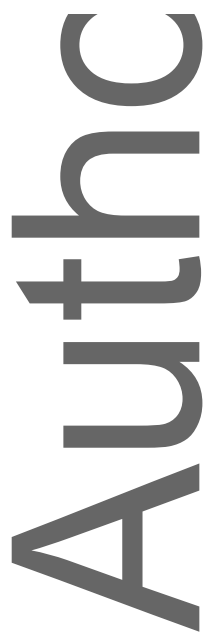




\section{University Library}

\section{- M I N E R VA \\ A gateway to Melbourne's research publications}

Minerva Access is the Institutional Repository of The University of Melbourne

Author/s:

Tiu, C;Wong, A;Herschtal, A;Mileshkin, L

Title:

Outcomes of Australian patients receiving non-funded anti-PD-1 immune checkpoint inhibitors for non-melanoma cancers

Date:

2018-08-01

\section{Citation:}

Tiu, C., Wong, A., Herschtal, A. \& Mileshkin, L. (2018). Outcomes of Australian patients receiving non-funded anti-PD-1 immune checkpoint inhibitors for non-melanoma cancers. ASIA-PACIFIC JOURNAL OF CLINICAL ONCOLOGY, 14 (4), pp.337-342. https:// doi.org/10.1111/ajco.12867.

Persistent Link:

http://hdl.handle.net/11343/283754 